\title{
SMOOTH MODEL OF SYSTEM WITH DIODE NONLINEARITY
}

\author{
Nguyen Thi Hien
}

Received: 12 September 2012 / Revised: 4 January 2013 / Accepted: 1 March 2013 /

Published online: 15 October 2013

(C) The Author(s) 2013. This article is published with open access at Springerlink.com

\begin{abstract}
A smooth model of a system with diode nonlinearity following the ordinary differential equation with a large parameter $K$ is proposed. It offers a convenient tool for numerical analysis using the advanced packages of the applied programs. The main result of this paper is presented in a theorem that gives the upper bound of the modeling error and shows that the model solution uniformly converges to the exact solution of system with diode nonlinearity when $K$ tends to infinity. The usefulness of this model is illustrated via a concrete example.
\end{abstract}

Keywords System with diode nonlinearity $\cdot$ Smooth model $\cdot$ External normal $\cdot$ Tangent and normal cones

Mathematics Subject Classification (2000) 34B60 - 34A40 · 93C15

\section{Introduction}

A rich set of applied mathematical problems with hysteresis nonlinearity such as nonlinear circuits, biological systems, automatic control systems, have been the target of many studies during the last century; but the direct mathematical modeling is dated only to 1960 . The Russian school under the supervision of Krasnoselskii has been doing intensively research along this line [3-5]. The principal results obtained by this group was presented in a Russian monograph published in 1983 and translated into English in 1989 [6]. Many articles and monographs were dedicated to different issues which relate to hysteresis nonlinearities (see $[1,8,10,11,14-16,18,19]$ and [12]). In this paper we consider one of the hysteresis nonlinearities, which is called a system with diode nonlinearity. The system with diode nonlinearity is studied as a mathematical description of electrical circuits with diode current transducers (see $[7,13,17]$ and [9]). Suppose that $Q \subset \mathbb{R}^{n}$ is a non-empty closed convex

N.T. Hien $(\bowtie)$

University of Transport and Communications, Cau Giay, Lang Thuong, Dong Da, Hanoi, Vietnam e-mail: hienvp@yahoo.com

e-mail: hienvp@mail.ru 
set; then the generalized system with diode nonlinearity has the following form (see [2] and [13]):

$$
\dot{x}=\tau_{x}(t, x),
$$

where $\tau_{x} f(t, x)$ is the projection of the vector $f(t, x)$ on the tangent cone $T_{Q}(x)$ of $Q$ at $x$ (see the following definition).

Definition Let $Q \subset \mathbb{R}^{n}$ be a convex set and $x \in Q$.

(i) The set

$$
N_{Q}(x)=\left\{\bar{x} \in \mathbb{R}^{n}:(\bar{x}, y-x) \leq 0 \forall y \in Q\right\}
$$

is called the normal cone of $Q$ at $x$.

(ii) The set

$$
N_{Q}^{*}(x)=\left\{\bar{x} \in \mathbb{R}^{n}:(\bar{x}, y) \leq 0 \forall y \in N_{Q}(x)\right\}
$$

is called the conjugate cone to the cone $N_{Q}(x)$ of $Q$ at $x$.

(iii) The set $T_{Q}(x)=N_{Q}^{*}(x)$ is called the tangent cone of $Q$ at $x$.

Assume that for $t \in\left[t_{0}, t_{0}+T\right]$ and $x \in Q$ the function $f(t, x)$ is continuous in the first argument $t$ and bounded by constant $C$, that is, $\|f(t, x)\| \leq C$; furthermore, $f(t, x)$ satisfies the Lipschitz condition in the second argument $x$ with the Lipschitz constant $L$. The solution of Eq. (1) is understood as a locally absolutely continuous function which satisfies (1) almost everywhere (see [2]); moreover, in [13] and [18] the authors have shown that the solution of (1) exists and is unique.

Obviously, the function on the right hand side of Eq. (1) can be discontinuous on the boundary of $Q$. Due to this discontinuity, it is impossible to solve (1) numerically. In order to study the behavior of the solution, it is necessary to transform (1) into a differential equation with a continuous right hand side. This paper presents such a technique named "smooth model" for which, we can obtain a numerical solution with any desired precision. Based on Eq. (1), the smooth model with diode nonlinearity is defined by the following equation:

$$
\dot{y}=f(t, \bar{y})-K(y-\bar{y}),
$$

where $\bar{y}=P_{Q}(y)$ is the projection of a point $y$ on the set $Q$ and $K$ is a large parameter.

The aim of this paper is presented in a theorem that gives the upper bound of the modeling error and shows that the model solution of (2) uniformly converges to the exact solution of (1) when $K$ tends to infinity.

\section{Lemmas}

\subsection{Lemma on projection}

Lemma $1 \bar{y}$ is the projection of $y$ on the non-empty closed convex set $Q \subset \mathbb{R}^{n}$ if and only if $\bar{y}$ is the unique element in $Q$ such that $(y-\bar{y}, x-\bar{y}) \leq 0$ for all $x \in Q$. That is,

$$
\left(\bar{y}=P_{Q}(y)\right) \quad \Longleftrightarrow(\exists ! \bar{y} \in Q \text { and }(y-\bar{y}, x-\bar{y}) \leq 0 \forall x \in Q) .
$$


Proof First, we prove that

$$
\left(\bar{y}=P_{Q}(y)\right) \Longrightarrow(\exists ! \bar{y} \in Q \text { and }(y-\bar{y}, x-\bar{y}) \leq 0 \forall x \in Q) .
$$

Obviously, it follows directly from $\bar{y}=P_{Q}(y)$ that $\bar{y} \in Q$. To prove the other expressions in the right hand side of (3) we put

$$
z(t)=t x+(1-t) \bar{y} \quad \forall x \in Q \text { and } t \in[0,1] .
$$

Now, we consider the function $\varphi(t)=\|y(t)-z(t)\|^{2}$. One can easily see that $\varphi(t)$ has minimum at $t=0$, then $\dot{\varphi}(0) \geq 0$. On the other hand, we have

$$
\begin{aligned}
\dot{\varphi}(t) & =2(y(t)-z(t), \dot{y}(t)-\dot{z}(t))=2(y-t x-(1-t) \bar{y}, \bar{y}-x) \\
& =-2(y-\bar{y}, x-\bar{y})+2 t\|x-\bar{y}\|^{2} .
\end{aligned}
$$

Thus, $\dot{\varphi}(0)=-2(y-\bar{y}, x-\bar{y}) \geq 0$, and, consequently, $(y-\bar{y}, x-\bar{y}) \leq 0$. Using the inequality $(y-\bar{y}, x-\bar{y}) \leq 0 \forall x \in Q$ we prove the uniqueness of the projection $\bar{y}$. Assume that there exist $\bar{y}_{1}, \bar{y}_{2}$ in $Q$ such that

$$
\left(y-\bar{y}_{1}, \bar{y}_{2}-\bar{y}_{1}\right) \leq 0 \quad \text { and } \quad\left(y-\bar{y}_{2}, \bar{y}_{1}-\bar{y}_{2}\right) \leq 0 .
$$

We have

$$
\left(y-\bar{y}_{1}, \bar{y}_{2}-\bar{y}_{1}\right)=-\left(y-\bar{y}_{2}, \bar{y}_{1}-\bar{y}_{2}\right)+\left(\bar{y}_{2}-\bar{y}_{1}, \bar{y}_{2}-\bar{y}_{1}\right) .
$$

It follows directly from (4) and (5) that $\bar{y}_{1}=\bar{y}_{2}$.

Second, we prove that

$$
\left(\bar{y}=P_{Q}(y)\right) \Longleftarrow(\exists ! \bar{y} \in Q \text { and }(y-\bar{y}, x-\bar{y}) \leq 0 \forall x \in Q) .
$$

By virtue of expressions in the right hand side of (3) we obtain $\bar{y} \in Q$ and $\|y-\bar{y}\| \leq$ $\|y-x\|$ for all $x \in Q$. It follows immediately from this fact that $\bar{y}=P_{Q}(y)$. The proof of the lemma is complete.

\subsection{Lemma on removing solution}

Lemma 2 For every solution y of $E q$. (2) and $\bar{y}=P_{Q}(y)$ we have

$$
\|y-\bar{y}\| \leq \frac{C}{K} .
$$

Proof Let $\varphi(t)=\|y(t)-\bar{y}(t)\|^{2}$. Then

$$
\begin{aligned}
\dot{\varphi}(t) & =2(y-\bar{y}, \dot{y}-\dot{\bar{y}})=2(y-\bar{y}, f(t, \bar{y})-K(y-\bar{y})-\dot{\bar{y}}) \\
& =2(y-\bar{y}, f(t, \bar{y}))-2 K\|y-\bar{y}\|^{2}-2(y-\bar{y}, \dot{\bar{y}}) .
\end{aligned}
$$

We will prove that $(y-\bar{y}, \dot{\bar{y}})=0$. Suppose the contrary, then there exists $t_{1} \in\left[t_{0}, t_{0}+T\right]$ such that

$$
\left(y\left(t_{1}\right)-\bar{y}\left(t_{1}\right), \dot{\bar{y}}\left(t_{1}\right)\right)=\alpha \neq 0 .
$$


If $\alpha>0$, then for sufficiently small value $\Delta t>0$ we have

$$
\bar{y}\left(t_{1}+\Delta t\right)-\bar{y}\left(t_{1}\right)=\dot{\bar{y}}\left(t_{1}\right) \cdot \Delta t+o(\Delta t) .
$$

By virtue of this fact and Eq. (8) we get

$$
\left(y\left(t_{1}\right)-\bar{y}\left(t_{1}\right), \frac{\bar{y}\left(t_{1}+\Delta t\right)-\bar{y}\left(t_{1}\right)}{\Delta t}\right)=\alpha+\left(y\left(t_{1}\right)-\bar{y}\left(t_{1}\right), \frac{o(\Delta t)}{\Delta t}\right) .
$$

Let $\Delta t \rightarrow 0$, then the right hand side of Eq. (9) tends to $\alpha>0$, and the left hand side is less than 0 , because $y\left(t_{1}\right)-\bar{y}\left(t_{1}\right) \in N_{Q}\left(\bar{y}\left(t_{1}\right)\right.$ ) (see the definition of the normal cone in Sect. 1). This immediately leads to a contradiction.

If $\alpha<0$, then for sufficiently small value $\Delta t<0$ we have

$$
\bar{y}\left(t_{1}+\Delta t\right)-\bar{y}\left(t_{1}\right)=\left(-\dot{\bar{y}}\left(t_{1}\right)\right) \cdot(-\Delta t)+o(\Delta t) .
$$

Consequently

$$
\left(y\left(t_{1}\right)-\bar{y}\left(t_{1}\right), \frac{\bar{y}\left(t_{1}+\Delta t\right)-\bar{y}\left(t_{1}\right)}{-\Delta t}\right)=-\alpha+\left(y\left(t_{1}\right)-\bar{y}\left(t_{1}\right), \frac{o(\Delta t)}{-\Delta t}\right) .
$$

Now, similarly to the case where $\alpha>0$, we can obtain a contradiction. Thus, $(y-\bar{y}$, $\dot{\bar{y}})=0$. It follows from this fact and Eq. (7) that

$$
\dot{\varphi}(t)=2(y-\bar{y}, f(t, \bar{y}))-2 K\|y-\bar{y}\|^{2} .
$$

By virtue of boundedness of $f$ we have

$$
\dot{\varphi} \leq 2 C \sqrt{\varphi}-2 K \varphi \text {. }
$$

Let $u(t)=\sqrt{\varphi(t)}$, then from Eq. (10):

$$
\dot{u} \leq C-K u \text {. }
$$

One can easily see that, if $u(t)$ is a solution of the inequality (11), then this solution also satisfies the following non-homogeneous equation:

$$
\dot{u}=-K u+C-b(t),
$$

where $b(t)$ is an arbitrary non-negative continuous function on $\left[t_{0}, t_{0}+T\right]$.

With the initial condition $u\left(t_{0}\right)=\sqrt{\varphi\left(t_{0}\right)}=0$, the solution of Eq. (12) is formulated as follows:

$$
u(t)=\int_{t_{0}}^{t} e^{K(s-t)}[C-b(s)] d s .
$$

It follows from this fact that

$$
\|y-\bar{y}\|=\|u\| \leq C \int_{t_{0}}^{t} e^{K(s-t)} d s \leq \frac{C}{K}
$$

for all $t \in\left[t_{0}, t_{0}+T\right]$, which completes the proof of the lemma. 


\section{Proximity theorem}

Theorem Suppose that $x(t), y(t)$ are solutions of systems (1) and (2), respectively, satisfying

$$
x\left(t_{0}\right)=y\left(t_{0}\right)=x_{0} \in Q .
$$

Then for all $t \in\left[t_{0}, t_{0}+T\right]$ the absolute error is bounded by

$$
\|x(t)-y(t)\| \leq \frac{C e^{L T}}{\sqrt{L}} \cdot \frac{1}{\sqrt{K}} .
$$

Proof For all $z \in \mathbb{R}^{n}$ we designate that $\eta_{x} z$ is the projection of $z$ on $N_{Q}(x)$. Then

$$
z=\tau_{x} z+\eta_{x} z
$$

(see [6]). It follows from this fact that Eq. (1) is equivalent to the following equation:

$$
\dot{x}=f(t, x)-\eta_{x} f(t, x) \text {. }
$$

Now, we estimate the value $p(t)=\frac{1}{2} \frac{d}{d t}\|x(t)-y(t)\|^{2}$. We have

$$
p(t)=(\dot{x}-\dot{y}, x-y)=\left(f(t, x)-f(t, \bar{y})-\eta_{x} f(t, x)+K(y-\bar{y}), x-y\right),
$$

and, consequently,

$$
\begin{aligned}
p(t)= & (f(t, x)-f(t, \bar{y}), x-y)+\left(\eta_{x} f(t, x), y-\bar{y}\right) \\
& +\left(\eta_{x} f(t, x), \bar{y}-x\right)+K(y-\bar{y}, x-\bar{y})+K(y-\bar{y}, \bar{y}-y) .
\end{aligned}
$$

Obviously, the last term of Eq. (14) is not positive; the inequalities $\left(\eta_{x} f(t, x), \bar{y}-x\right) \leq 0$ and $(y-\bar{y}, x-\bar{y}) \leq 0$ follow directly from the definition of the normal cone and the lemma on projection, respectively. Therefore, by virtue of (14) we obtain

$$
p(t) \leq(f(t, x)-f(t, \bar{y}), x-y)+\left(\eta_{x} f(t, x), y-\bar{y}\right) .
$$

To estimate the value $p(t)$ we estimate every term in the right hand side of the inequality (15). From the lemma on removing solution and the boundedness of $f$ it follows that

$$
\left\|\left(\eta_{x} f(t, x), y-\bar{y}\right)\right\| \leq \frac{C^{2}}{K} .
$$

On the other hand, by virtue of the Lipschitz condition of function $f(t, x)$ in the second argument we get

$$
\|(f(t, x)-f(t, \bar{y}), x-y)\| \leq L\|x-\bar{y}\| \cdot\|x-y\| .
$$

It is easy to see that $\|x-\bar{y}\| \leq\|x-y\|$. Thus,

$$
\|(f(t, x)-f(t, \bar{y}), x-y)\| \leq L\|x-y\|^{2} .
$$

From the inequalities (15)-(17) it follows that

$$
p(t) \leq L\|x-y\|^{2}+\frac{C^{2}}{K} .
$$


From the last inequality it follows that the absolutely continuous function $u(t)=\| x(t)-$ $y(t) \|^{2}$ satisfies the linear non-homogeneous equation

$$
\dot{u}=2 L u+2 \frac{C^{2}}{K}-b(t),
$$

where $b(t)$ is an arbitrary non-negative continuous function on $\left[t_{0}, t_{0}+T\right]$. With the initial condition $u\left(t_{0}\right)=0$ we can obtain

$$
u(t)=2 \int_{t_{0}}^{t} e^{2 L(t-s)} \cdot\left[\frac{C^{2}}{K}-\frac{b(s)}{2}\right] d s .
$$

Consequently, for $t \in\left[t_{0}, t_{0}+T\right]$ we have

$$
\|x(t)-y(t)\|^{2} \leq \frac{2 C^{2}}{K} \int_{t_{0}}^{t} e^{2 L(t-s)} d s \leq \frac{C^{2}}{K L} e^{2 L T} .
$$

From this fact the inequality (13) easily follows, which completes the proof of the theorem.

\section{Example}

We consider the electrical circuit of Fig. 1 known as a full-wave rectifier; it contains four diodes, a source, resistance $R$ and inductance $L_{0}$. This example is used to show the system with diode nonlinearity of the form (1) in one hand, and on the other to show the numerical technique to obtain its solution model by using the smooth model.

\subsection{Mathematical description for the circuit}

Diodes shall be ideal, that is, their current $i_{k}$ and voltage $u_{k}$ from the anode to the cathode satisfy the following system:

$$
\left\{\begin{array}{l}
i_{k} \geq 0 \\
u_{k} \leq 0 \\
i_{k} u_{k}=0
\end{array}\right.
$$

In the supply circuit there is the source which contains a voltage $e(t)$, resistance $r$, and inductance $l_{0}$. The choice of positive voltage is marked by indicators in the Fig. 1 . We denote

Fig. 1 The full-wave rectifier

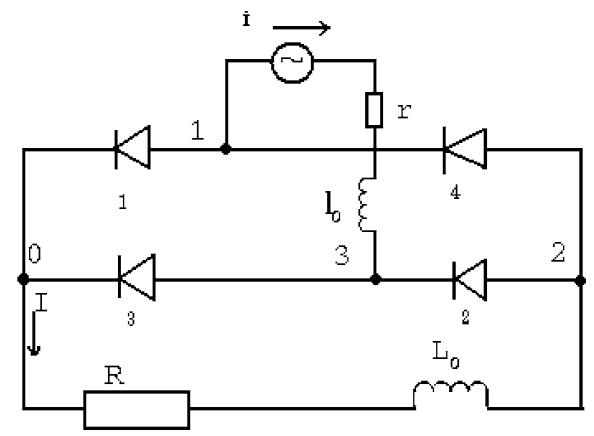


the input voltage of the supply circuit (between nodes 1 and 3) by $u_{1}$ and the voltage of the load circuit (between nodes 2 and 0 ) by $u_{2}$. Using the sign convention of the circuit theory, the Kirchhoff laws applied to this circuit give the following equations:

$$
\left\{\begin{array}{l}
u_{l_{0}}+r i+u_{1}=e(t) \\
u_{L_{0}}+R I+u_{2}=0
\end{array}\right.
$$

We also have $u_{l_{0}}=l_{0} \cdot \frac{d i}{d t}$ and $u_{L_{0}}=L_{0} \cdot \frac{d I}{d t}$, so the system (18) can be rewritten as follows:

$$
\left\{\begin{array}{l}
l_{0} \cdot \frac{d i}{d t}+r i+u_{1}=e(t) \\
L_{0} \cdot \frac{d I}{d t}+R I+u_{2}=0
\end{array}\right.
$$

We use the following changes of variables:

$$
x=\left(\begin{array}{l}
x_{1} \\
x_{2}
\end{array}\right)=\left(\begin{array}{c}
i \sqrt{l_{0}} \\
I \sqrt{L_{0}}
\end{array}\right) \quad \text { and } \quad z=\left(\begin{array}{c}
z_{1} \\
z_{2}
\end{array}\right)=\left(\begin{array}{c}
\frac{u_{1}}{\sqrt{l_{0}}} \\
\frac{u_{2}}{\sqrt{L_{0}}}
\end{array}\right) .
$$

Then the system (19) is defined as follows:

$$
\dot{x}+A x+z=E(t)
$$

where

$$
A=\left(\begin{array}{cc}
\frac{r}{l_{0}} & 0 \\
0 & \frac{R}{L_{0}}
\end{array}\right) \quad \text { and } \quad E(t)=\left(\begin{array}{c}
\frac{e(t)}{\sqrt{l_{0}}} \\
0
\end{array}\right) .
$$

We follow [13] to prove that $x$ lies in the cone $Q$ which is the conical hull of the vectors

$$
\left(\begin{array}{c}
\sqrt{\frac{l_{0}}{L_{0}}} \\
1
\end{array}\right) \quad \text { and } \quad\left(\begin{array}{c}
-\sqrt{\frac{l_{0}}{L_{0}}} \\
1
\end{array}\right)
$$

and $z$ lies in the normal cone $N_{Q}(x)$ of this cone $Q$ at $x$. If we denote $g(t, x)=E(t)-A x$, then equation (20) is equivalent to the following equation (see [2]):

$$
\dot{x}=\tau_{x} g(t, x) \text {. }
$$

One can easily see that the function $g(t, x)$ for $t \in\left[t_{0}, t_{0}+T\right]$ and $x \in Q$ satisfies all the conditions stated in Sect. 1.

\subsection{Behavior of solution}

For the numerical analysis of the system (21) we use the studied smooth model of the form (2); for this circuit, it becomes

$$
\dot{y}=g(t, \bar{y})-K(y-\bar{y}),
$$

where $\bar{y}=P_{Q}(y)$ and $K$ is a large parameter. 
Fig. 2 The trajectory of the smooth model solution for $K$ sufficiently large
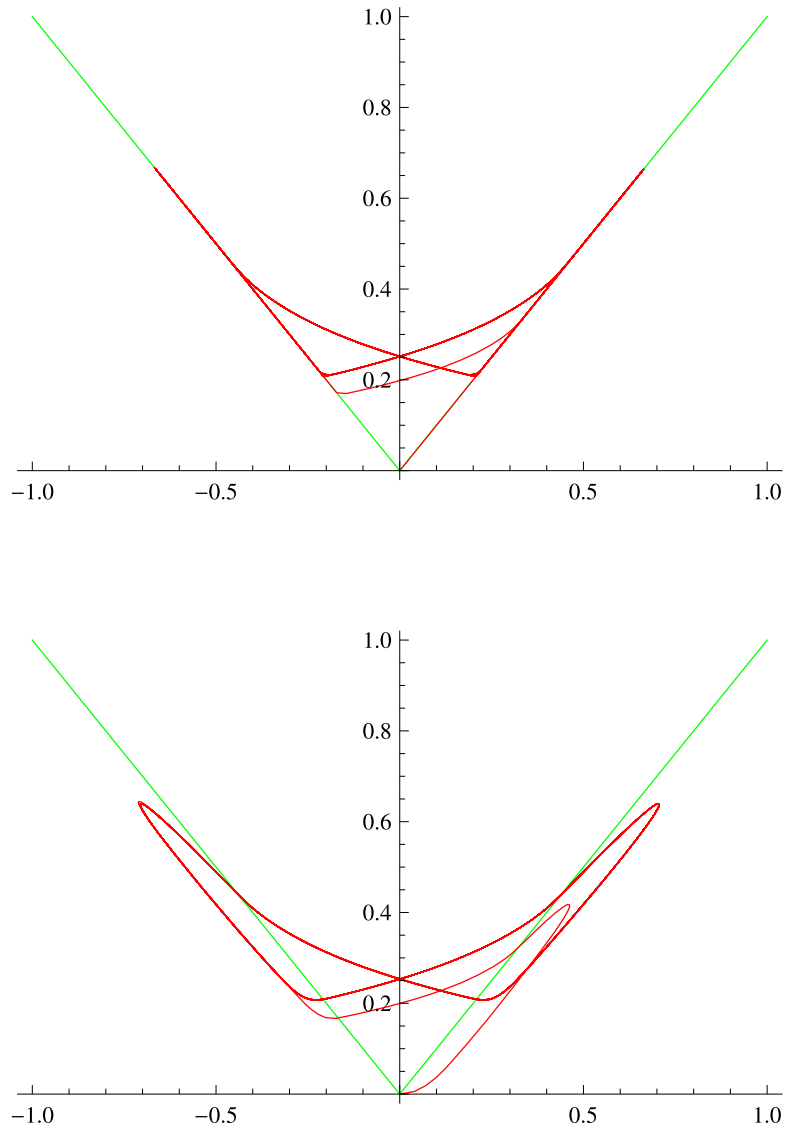

$-1.0$
Fig. 3 The trajectory of the smooth model solution for $K$ sufficiently small

Using the program Mathematica 7.0, we obtained some numerical results for the state trajectory of (22), which is approximated to the state trajectory of (21), given the following parameters and initial conditions:

$$
l_{0}=L_{0}=1 ; \quad r=0.1 ; \quad R=0.2 ; \quad e(t)=3 \cos 2 t ; \quad t_{0}=0 ; \quad T=5 \text { and } y_{0}=\left(\begin{array}{l}
0 \\
0
\end{array}\right)
$$

For $K=K_{1}=10^{5}$, and $K=\frac{K_{1}}{2}$, the two corresponding trajectories are impossible to be distinguished as shown in Fig. 2

This means that the proposed smooth model of form (2) gives a consistent result, which does not noticeably depend on parameter $K$ for $K$ sufficiently large.

When the value $K$ is chosen small, for example, $K=10$, the obtained result shows that the smooth model (2) does not give consistent results; in fact, its trajectory does not completely lie in the cone $Q$ (see Fig. 3) and it strongly depends on the value $K$.

Thus, the proposed smooth model (2) for the sufficiently large value $K$ can be numerically solved in view of analyzing the behavior of the system with diode nonlinearity (1). 


\subsection{Error of the smooth model solution}

If $x(t), y(t)$ are, respectively, solutions of systems (21) and (22), then by virtue of the proximity theorem, we obtain for $t \in\left[t_{0}, t_{0}+T\right]$ the following estimate:

$$
\|x(t)-y(t)\| \leq \frac{C e^{L T}}{\sqrt{L}} \cdot \frac{1}{\sqrt{K}} .
$$

Suppose that the parameters and the initial conditions are described in (23), the constants $C$ and $L$ are calculated as follows. First, the Lipschitz constant of the function $g(t, x)$ is

$$
L=\|A\|=\left\|\left(\begin{array}{cc}
\frac{r}{l_{0}} & 0 \\
0 & \frac{R}{L_{0}}
\end{array}\right)\right\|=\max \left\{\frac{r}{l_{0}}, \frac{R}{L_{0}}\right\}=0.2 .
$$

Second, for the sufficiently large value $K$ the behavior of solution of (21) is shown in Fig. 2, consequently, $\|x\| \leq \sqrt{2}$ for $t \in[0,10]$; and then

$$
\|f(t, x)\| \leq\|E(t)\|+\|A\| \cdot\|x\| \leq 3+0.2 \sqrt{2}=C .
$$

Thus, the error of the solutions of (21) and (22) satisfies the following estimate:

$$
\|x(t)-y(t)\| \leq \frac{\sqrt{5}(3+0.2 \sqrt{2}) e}{\sqrt{K}} .
$$

From this bound it directly follows that for the sufficiently large $K$ this error can be chosen as small as desired.

\section{Conclusions}

This paper proposes a smooth model to approximate the system with diode nonlinearity which is called the exact model. This proposed model explicitly contains a parameter $K$. It is shown that the proposed smooth model uniformly converges to the exact model when $K$ tends to infinity; this means that for very large $K$, there is no difference between the exact solution and the smooth model solution; we can therefore adjust $K$ to ensure any desired precision for which, the smooth model can be considered as equivalent to the exact model. Finally, this smooth model can be effectively used to analyze the solution behavior or for simulation on computers of systems with diode nonlinearity.

Open Access This article is distributed under the terms of the Creative Commons Attribution License which permits any use, distribution, and reproduction in any medium, provided the original author(s) and the source are credited.

\section{References}

1. Appell, J., Pryadko, I.N., Sadovsky, B.N.: On the stability of some relay-type regulation system. Z. Angew. Math. Mech. 88(10), 808-816 (2008)

2. Filippov, A.F.: Differential Equations with Discontinuous Right Hand Sides. Kluwer Academic, Norwell (1988)

3. Krasnosel'skii, M.A., Pokrovskii, A.V.: Periodic oscillations in systems with relay nonlinearities. J. Sov. Math. 3, 873-877 (1974) 
4. Krasnosel'skii, M.A., Pokrovskii, A.V.: Simulation of transformers with hysteresis by means of continuum of relay nonlinearities. J. Sov. Math. 2, 447-451 (1976)

5. Krasnosel'skii, M.A., Pokrovskii, A.V., Klepcyn, A.F., Livshitz, E.A., Vladimirov, A.A.: Vector hysteresis nonlinearities of the von Mizes-Tresca type. Sov. Phys. Dokl. 3, 581-583 (1981)

6. Krasnosel'skii, M.A., Pokrovskii, A.V.: Systems with Hysteresis. Springer, Berlin (1989)

7. Mathanov, P.N.: Basic Analysis of Electrical Circuits. Linear Chain. High School, Moscow (1981)

8. Mayergoyz, I.D.: Mathematical Models of Hysteresis. Springer, Berlin (1991)

9. Nesterenko, R.V., Sadovskii, B.N.: Forced oscillations in a two-dimensional cone. Autom. Remote Control 63(2), 181-188 (2002)

10. Hien, N.T.: Analysis of autooscillation in system with two relays. Works of the Mathematical faculty, Voronezh VGU vol. 10, pp. 112-118 (2006)

11. Hien, N.T.: Smooth model of a support and gap. Vestn. VGU 2, 92-95 (2009)

12. Hien, N.T., Sadovskii, B.N.: Smooth model of the relay with hysteresis. Autom. Remote Control 71(11), 2320-2330 (2010)

13. Petrova, L.P., Sadovskii, B.N.: A Mathematical Theory of Electrical Circuits with Diode Current Transducers, Voronezh (1982)

14. Pokrovskii, A.V., Brokate, M.: Asymptotically stable periodic oscillation in systems with hysteresis nonlinearities. J. Differ. Equ. 150, 98-123 (1998)

15. Pryadko, I.N., Sadovskii, B.N.: On locally explicit models of some non-smooth systems. Autom. Remote Control 65, 1556-1565 (2004)

16. Pryadko, I.N., Sadovskii, B.N.: On locally explicit equations and systems with switching. Funct. Differ. Equ. 13, 571-584 (2006)

17. Sadovskii, B.N., Sobolev, M.P.: A mathematical theory of circuits with thyristors. Collection of scientific papers. Dynamics of heterogeneous systems. Moscow, VNII-SI, 178-182 (1984)

18. Sadovskii, B.N.: Notes on systems with diode nonlinearity. Univ. Calabria, Dip. Mat., Cosenza, Italy (1988)

19. Tsipkin, J.Z.: Automatic Relay Systems. Nauka, Moscow (1974) 